Published in final edited form as:

Int J Cardiol. 2015 November 1; 198: 222-228. doi:10.1016/j.ijcard.2015.06.093.

\title{
Application and Outcomes of a Hybrid Approach to Chronic Total Occlusion Percutaneous Coronary Intervention in a Contemporary Multicenter US Registry
}

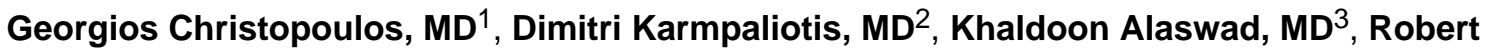 \\ W. Yeh, MD ${ }^{4}$, Farouc A. Jaffer, MD, PhD $^{5}$, Wyman R. Michael, MD $^{5}$, William Lombardi, MD $^{6}$, \\ Rohan V. Menon, BS ${ }^{1}$, Aaron Grantham, MD, David Kandzari, MD ${ }^{8}$, Nicholas Lembo, MD $^{8}$, \\ Jeffrey W. Moses, MD², Ajay Kirtane, MD², Manish Parikh, MD², Philip Green, MD², Matthew \\ Finn, MD², Santiago Garcia, MD ${ }^{9}$, Anthony Doing, MD $^{10}$, Mitul Patel, MD ${ }^{11}$, John \\ Bahadorani, MD ${ }^{11}$, Muhammad Nauman J. Tarar, MD1 , Georgios E. Christakopoulos, MD1, \\ Craig A. Thompson, MD, MMSc ${ }^{12}$, Subhash Banerjee, MD ${ }^{1}$, and Emmanouil S. Brilakis, MD, \\ $\mathrm{PhD}^{1}$
}

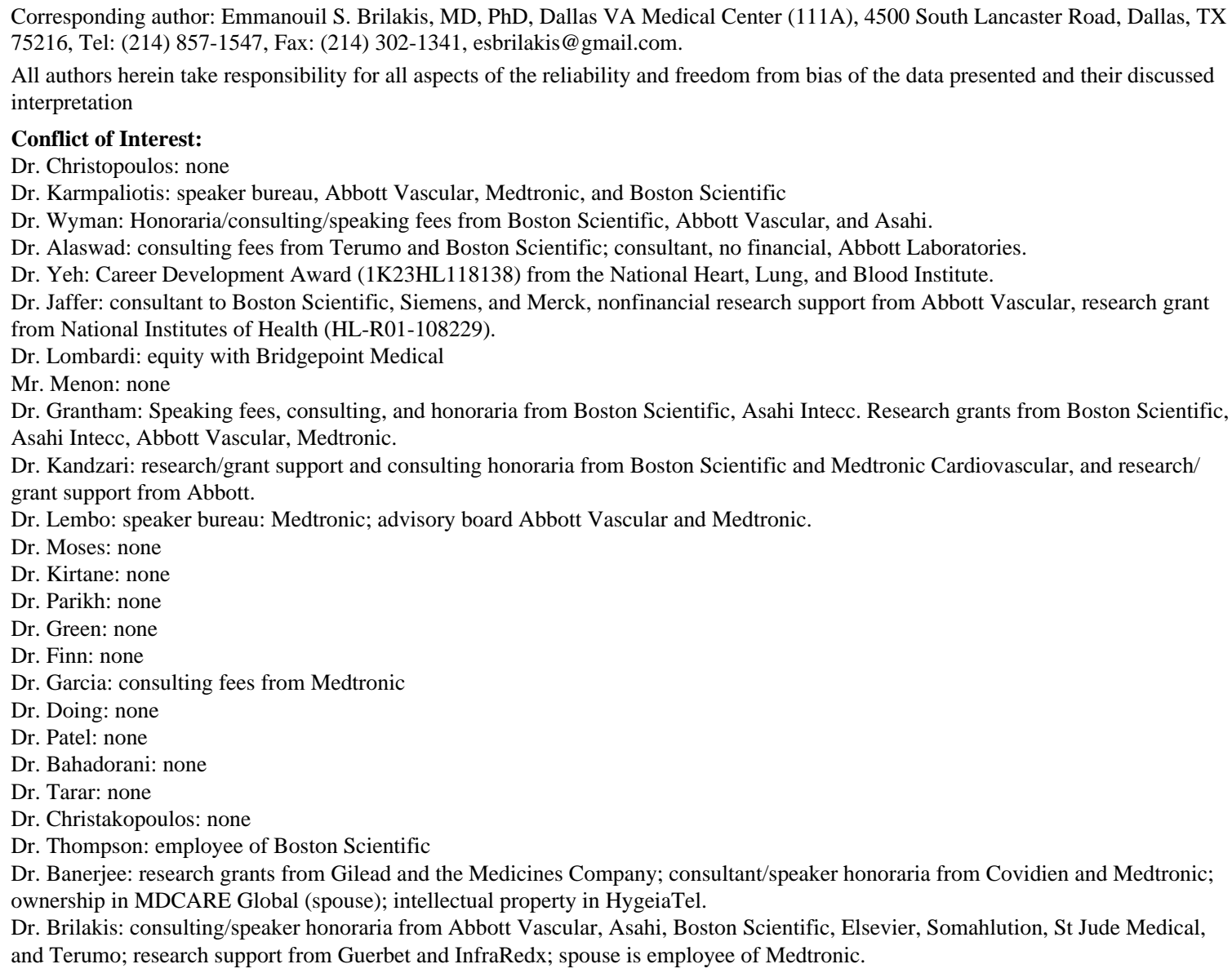

Publisher's Disclaimer: This is a PDF file of an unedited manuscript that has been accepted for publication. As a service to our customers we are providing this early version of the manuscript. The manuscript will undergo copyediting, typesetting, and review of the resulting proof before it is published in its final citable form. Please note that during the production process errors may be discovered which could affect the content, and all legal disclaimers that apply to the journal pertain. 
1VA North Texas Healthcare System and UT Southwestern Medical Center, Dallas, TX

${ }^{2}$ Columbia University, New York, NY

${ }^{3}$ Henry Ford Hospital, Detroit, MI

${ }^{4}$ Massachusetts General Hospital and Harvard Medical School, Boston, MA

${ }^{5}$ Torrance Memorial Medical Center, Torrance, CA

${ }^{6}$ University of Washington, Seattle, WA

${ }^{7}$ Mid America Heart Institute, Kansas City, MO

${ }^{8}$ Piedmont Heart Institute, Atlanta, GA

${ }^{9}$ Minneapolis VA Healthcare System and University of Minnesota, Minneapolis, MN

${ }^{10}$ Medical Center of the Rockies, Loveland, CO

${ }^{11}$ VA San Diego Healthcare System and University of California San Diego, San Diego, CA

${ }^{12}$ Boston Scientific, Natick, MA

\section{Abstract}

Background-A hybrid approach to chronic total occlusion (CTO) percutaneous coronary intervention (PCI) prioritizing and combining all available crossing techniques was developed to optimize procedural efficacy, efficiency, and safety, but there is limited published data on its outcomes.

Methods-We examined the procedural techniques and outcomes of 1,036 consecutive CTO PCIs performed using a hybrid approach between 2012 and 2015 at 11 US centers.

Results-Mean age was $65 \pm 10$ years and $86 \%$ of the patients were men, with a high prevalence of diabetes mellitus (43\%) and prior coronary artery bypass graft surgery (34\%). Most target CTOs were located in the right coronary artery (59\%), followed by the left anterior descending artery (23\%) and the circumflex (19\%). Dual injection was used in $71 \%$. Technical success was achieved in $91 \%$ and a major procedural complication occurred in $1.7 \%$ of cases. The final successful crossing technique was antegrade wire escalation in $46 \%$, antegrade dissection/re-entry in $26 \%$, and retrograde in $28 \%$. The initial crossing strategy was successful in $58 \%$ of the lesions, whereas $39 \%$ required an additional approach. Overall, antegrade wire escalation was used in $71 \%$, antegrade dissection/re-entry in 36\%, and the retrograde approach in $42 \%$ of procedures. Median contrast volume, fluoroscopy time, and air kerma radiation dose were 260 (200-360) ml, 44 (27-72) min, and 3.4 (2.0-5.4) Gray, respectively.

Conclusion-Application of a hybrid approach to CTO crossing resulted in high success and low complication rates across a varied group of operators and hospital practice structures, supporting its expanding use in CTO PCI.

\section{Keywords}

chronic total occlusion; percutaneous coronary intervention; techniques; outcomes 


\section{Introduction}

Successful percutaneous coronary intervention (PCI) of chronic total occlusions (CTOs) can provide significant clinical benefits (1-4). However, it can be challenging to perform, with inability to cross the occlusion being the main mode of failure (5). Three broad categories of CTO crossing techniques are currently utilized (6): antegrade wire escalation (7), antegrade dissection/re-entry (8-11), and the retrograde approach (12-14), all of which have contributed to improved procedural success rates (15-19). Selecting the optimal technique for each CTO lesion can be challenging, but this process has recently been streamlined with the introduction of a hybrid CTO crossing algorithm (Figure 1) (20). The hybrid algorithm assesses four key angiographic characteristics to determine the optimal initial and subsequent crossing strategies (20). The term hybrid refers to (a) sequential use of CTO crossing techniques if the initially attempted technique fails and (b) unification of all 3 currently available CTO crossing techniques under a single algorithm (19-26). The goal of the present study was to examine the impact of a hybrid approach on procedural workflow and outcomes of CTO PCI in a multicenter, contemporary CTO PCI registry (27).

\section{Methods}

\section{Patient population}

We examined the procedural techniques and outcomes of 1,036 consecutive CTO PCIs (1,019 patients) performed using a hybrid approach between January 2012 and March 2015 at 11 US centers: Appleton Cardiology, Appleton Wisconsin; Columbia University, New York, New York; Massachusetts General Hospital, Boston, Massachusetts; Medical Center of the Rockies, Loveland, Colorado; Piedmont Heart Institute, Atlanta Georgia; St. Joseph Medical Center, Bellingham Washington; St. Luke's Health System's Mid-America Heart Institute, Kansas City, Missouri; Torrance Memorial Center, Torrance, California; VA Minneapolis Healthcare System, Minneapolis, Minnesota, VA North Texas Health Care System, Dallas, Texas, and VA San Diego Healthcare System, San Diego, California. All procedures were performed by operators with expertise in CTO PCI using a hybrid approach. Data collection was performed both prospectively and retrospectively and recorded in a dedicated CTO database (PROGRESS CTO, Clinicaltrials.gov Identifier: NCT02061436). The study was approved by the institutional review board of each site.

\section{Definitions}

Coronary CTOs were defined as coronary lesions with thrombolysis in myocardial infarction (TIMI) grade 0 flow of at least 3 month duration. Estimation of the occlusion duration was based on first onset of anginal symptoms, prior history of myocardial infarction in the target vessel territory, or comparison with a prior angiogram.

Calcification was assessed by angiography as mild (spots), moderate (involving $\mathbf{5 0 \%}$ of the reference lesion diameter) and severe (involving $>50 \%$ of the reference lesion diameter). Moderate proximal vessel tortuosity was defined as the presence of at least 2 bends $>70$ degrees or 1 bend $>90$ degrees and severe tortuosity as 2 bends $>90$ degrees or 1 bend $>120$ degrees in the CTO vessel. Technical success of CTO PCI was defined as successful CTO 
revascularization with achievement of $<30 \%$ residual diameter stenosis within the treated segment and restoration of TIMI grade 3 antegrade flow. Procedural success was defined as achievement of technical success with no inhospital major adverse cardiac events (MACE). In-hospital MACE included any of the following adverse events prior to hospital discharge: death, acute myocardial infarction, urgent repeat target vessel revascularization with PCI or coronary artery bypass graft surgery (CABG), tamponade requiring either pericardiocentesis or surgery, and stroke. Major bleeding was defined as bleeding resulting in transfusion, requiring surgical intervention, or causing hemoglobin drop $23 \mathrm{~g} / \mathrm{dL}$.

\section{Statistical analysis}

Descriptive statistics were used to report the angiographic measurements, clinical characteristics, and in-hospital outcomes. All procedures were categorized based on technical success or technical failure and compared in terms of baseline clinical characteristics, angiographic characteristics and procedural data. Categorical variables were expressed as percentages and compared using the chi-square test or Fischer's exact test. Continuous variables were presented as mean \pm standard deviation or median (interquartile range) and were compared using the t-test, one-way analysis of variance or Wilcoxon ranksum test, as appropriate. All statistical analyses were performed with JMP 11.0 (SAS Institute, Cary, North Carolina). Two-sided $p$-values $<0.05$ were considered statistically significant.

\section{Results}

\section{Patient characteristics}

The mean age was $65 \pm 10$ years. Most patients (86\%) were men, with a high prevalence of diabetes mellitus (43\%), dyslipidemia (96\%), hypertension (90\%), peripheral arterial disease $(17 \%)$, prior PCI (67\%), and prior CABG (34\%) (Table 1). Compared with patients in whom CTO PCI was successful, patients in whom CTO PCI failed were more likely to have had a history of myocardial infarction ( $58 \%$ vs. $40 \%$, p=0.001), CABG ( $46 \%$ vs. $33 \%, 0.013$ ), PCI ( $78 \%$ vs. $65 \%, 0.015)$ or stroke ( $17 \%$ vs. $11 \%, \mathrm{p}=0.048)$.

\section{Angiographic and procedural characteristics}

The most common CTO target vessel was the right coronary artery (59\%), followed by the left anterior descending artery (23\%) and the circumflex (19\%). Moderate to severe calcification and moderate to severe tortuosity were present in $56 \%$ and $33 \%$ of the CTO lesions, respectively. PCI of the target CTO had been attempted in $16 \%$ of cases and $13 \%$ of

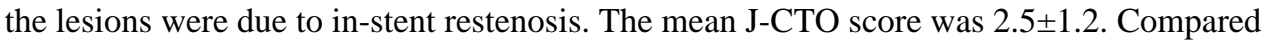
with successful procedures, CTO lesions in the failed procedures were longer (38 [25-51] vs. 30 [20-40] mm, $\mathrm{p}=0.014)$, and more calcified ( $68 \%$ vs. $54 \%, \mathrm{p}=0.008)$, and had greater tortuosity ( $45 \%$ vs. $32 \%, \mathrm{p}=0.010$ ), and higher $\mathrm{J}-\mathrm{CTO}$ scores ( $3.2 \pm 1.0$ vs. $2.4 \pm 1.2, \mathrm{p}<0.001$ ) (Table 1). Lack of "interventional" collaterals (suitable for the retrograde approach) was also more frequent in failed cases $(58 \%$ vs. $36 \%, \mathrm{p}<0.001)$. 


\section{Outcomes}

Technical success and procedural success were achieved in $91 \%$ and $90 \%$ of the procedures, respectively. Median total procedure time, fluoroscopy time, air kerma radiation exposure and contrast volume were 119 (82-175) $\min , 44$ (27-72) min, 3.4 (2.0-5.4) Gray, and 260 (200-360) $\mathrm{ml}$, respectively. An average of $2.5 \pm 1.1$ stents were implanted in $98 \%$ of the successful cases, and $98 \%$ of the stents were overlapping (Table 2).

A major complication occurred in 17 patients (1.7\%): 3 patients died (1 due to cardiac tamponade followed by unsuccessful pericardiocentesis, 1 due to myocardial infarction and 1 due to a vascular access complication), 8 patients had an acute myocardial infarction (one of whom died, as mentioned above), 1 patient experienced a stroke, 5 patients had cardiac tamponade requiring pericardiocentesis (one of whom died, as mentioned above), and 2 patients required urgent target vessel revascularization with PCI. Major bleeding occurred in 6 patients $(0.6 \%)$, vascular access complications in $15(1.4 \%)$, and donor vessel injury including perforation in 40 cases (3.9\%).

\section{CTO crossing strategies}

The type and sequence of CTO crossing techniques utilized in the study patients is presented in Table 3. Radial access was used in $22 \%$ and dual injection was utilized in $71 \%$ of the CTO lesions.

The initial crossing approach was antegrade wire escalation in 66\%, antegrade dissection/reentry in $14 \%$, and retrograde in $19 \%$ of the cases (Figure 2). The initial crossing approach was successful in 598 (58\%) procedures, while additional crossing strategies were utilized in 404 procedures (39\%). The remaining 34 cases (3\%) were unsuccessful and were stopped after the first crossing attempt. The success rates of antegrade wiring, antegrade dissection/re-entry, and retrograde crossing as initial approach was 55\%, 60\% and 66\%, respectively $(\mathrm{P}=0.671)$.

Overall, antegrade wire escalation was used in $71 \%$, antegrade dissection/reentry in $36 \%$, and the retrograde approach in $42 \%$. Among successful cases ( $n=940)$, the final successful crossing technique was antegrade wire escalation in $46 \%$, antegrade dissection/re-entry in $26 \%$, and retrograde in $28 \%$. An average of $1.5 \pm 0.8$ crossing strategies were utilized, with failed CTO PCI procedures requiring more approach changes than successful ones (Table 3). The clinical and angiographic characteristics of patients, classified according to the initial crossing strategy, are presented in Table 4.

The final technical success among cases in which the initial crossing approach was antegrade wire escalation, antegrade dissection/re-entry, and retrograde was 92\%, 93\%, and $86 \%$, respectively ( $\mathrm{P}=0.030$, Figure 2 ). Antegrade wiring was the most common initial technique, however dissection/re-entry and the retrograde approach were increasingly used later in the algorithm if the classic antegrade techniques failed (Figures 3A-C).

Retrograde crossing-The retrograde approach was used in 431 cases and was successful in $273(63 \%)$. Among successful retrograde cases, septal collaterals were used in $51 \%$, epicardial collaterals in $29 \%$ and saphenous vein grafts in $17 \%$ (more than one type of 
collateral was used in some cases). Internal mammary artery grafts and smaller collaterals were used in the remaining successful retrograde cases $(2 \%)$. The following retrograde crossing techniques were used: reverse controlled antegrade and retrograde tracking and dissection (reverse CART) (66\%), retrograde true lumen puncture (19\%), knuckle wire (6\%), and CART (4\%), and "just marker" technique (retrograde guidewire serving as marker of the distal true lumen) (3\%) (more than one crossing techniques were used in some cases). Among successful retrograde cases $(n=273)$, externalization was performed in $82 \%$.

Antegrade dissection/re-entry-Overall, antegrade dissection/re-entry was used in 368 patients and was successful in $243(66 \%)$. Among successful antegrade dissection/re-entry cases, a dissection strategy was used in $91 \%$ of the time. The dissection techniques utilized included: the CrossBoss catheter (63\%), knuckle wire (28\%), and subintimal guidewire entry (9\%). Subintimal crossing occurred in $74 \%$ and true lumen crossing in $26 \%$ of the cases. Use of the CrossBoss catheter resulted in true lumen crossing in $21 \%$ of patients. A re-entry method was utilized for $80 \%$ of subintimal crossings, and was successful in $81 \%$, as follows: Stingray balloon and guidewire (79\%), subintimal antegrade tracking and re-entry (STAR) (11\%), limited antegrade subintimal tracking (LAST) (3\%), or other wire based methods (7\%) (Table 5).

\section{Discussion}

Our study provides unique insight into the effectiveness and safety of a hybrid approach to CTO PCI among various centers and operators. Although success with the initial crossing strategy was $58 \%$, after use of additional strategies a final technical success rate of $91 \%$ was achieved with a low incidence of MACE (1.7\%).

The first step of the hybrid approach is dual injection to optimally view the CTO target vessel, estimate lesion length and evaluate the size and quality of the distal target vessel, allowing optimal initial crossing strategy selection $(23,28)$. Dual injection is performed via selective coronary angiography using both the CTO PCI target vessel and another vessel, such as the contralateral coronary artery or a bypass graft, that provides collaterals to the distal target vessel. Dual injection was utilized in $71 \%$ of our cases and is crucial for achieving high success rates and maximal safety in CTO PCI.

The selection of the optimal initial CTO crossing strategy is dependent on the anatomy of the coronary vessels. Four angiographic characteristics are used to make the decision (Figure 1): (a) lesion length (lesions $220 \mathrm{~mm}$ in length tend to have lower success rates and longer procedure times using standard antegrade wire escalation (23)); (b) proximal cap location and morphology; (c) size, quality (i.e. vessel size and presence of luminal stenoses and/or calcification) and presence of side branches of the target coronary vessel at the distal cap; and (d) size and suitability of collateral circulation for the retrograde technique (29).

In most patients in our series (66\%) antegrade wire escalation was selected as the initial crossing strategy and was successful in 55\%, whereas additional crossing strategies were necessary in $43 \%$ of the patients (Figure 2). Hence, the ability to switch crossing strategies is of vital importance to achieving high success rates. In fact, $64 \%$ of cases in our registry 
required an alternative approach to antegrade wire escalation to achieve successful crossing. Our study confirms that antegrade wire escalation remains the most commonly utilized CTO crossing technique and was the final successful technique in the highest proportion of successful CTO PCI cases (46\%). Therefore, solid antegrade wiring skills are critically important for all CTO operators.

With regards to specific CTO devices, the CrossBoss catheter and Stingray balloon and wire (Boston Scientific) (23) were used in the majority of the successful antegrade dissection/reentry cases. Importantly, in $21 \%$ of the procedures use of the CrossBoss catheter resulted in distal true lumen crossing, which is in line with the findings of the Facilitated Antegrade Steering Technique in Chronic Total Occlusions (FAST CTOs) trial. $(10,23,30)$ In cases of subintimal crossing successful true lumen reentry was achieved in most cases $(75 \%)$, and most re-entry attempts were performed using the dedicated Stingray system with infrequent use of wire-based re-entry techniques.

Epicardial collaterals were used in $29 \%$ of the successful retrograde crossing attempts, attesting to the experience of the operators in our series, as epicardial collateral crossing carries increased risk compared to septal collateral crossing (10). Similar to other contemporary CTO PCI series (31) reverse CART was the most commonly used technique for retrograde CTO crossing. Wire externalization was performed in $80 \%$ of the retrograde cases and snaring was required in $20 \%$ of these cases. Familiarity and experience with use of snares and snaring techniques is, therefore, important when the retrograde technique is utilized $(14,16)$.

Application of a hybrid approach resulted in high technical (91\%) and procedural (90\%) success in our series of 1,036 CTO PCIs. This is similar to the rates reported in prior studies. Nombela-Franco et al reported the outcomes 209 consecutive patients who underwent hybrid CTO PCI by a high-volume operator with $90.4 \%$ final angiographic success rate (25). Pershad et al. reviewed outcomes before and after adoption of a hybrid approach at two experienced CTO PCI centers (19). Among 660 cases (462 performed before and 198 performed after hybrid approach implementation), both technical (95.4\% vs. $79.4 \%$, $\mathrm{P}<0.001)$ and procedural $(88.3 \%$ vs. $77.9 \%, \mathrm{P}<0.001)$ success significantly improved. Antegrade wire escalation, antegrade dissection/reentry, and the retrograde approach were used in $39.3 \%, 28.7 \%$ and $35.3 \%$ of cases, respectively, with final success rate of $87.1 \%$, $94.7 \%$, and $78.8 \%$, respectively. Michael et al. described application of a hybrid approach in 73 consecutive CTO PCI cases performed by a single operator (26). Technical success was $90.4 \%$ and the incidence of complications was $4.1 \%$. The initial crossing approach succeeded in $54.8 \%$, and the crossing strategies used were similar to what was observed in our study. The above studies are in line with the results of the present study, which highlights that combining multiple crossing approaches during the same procedure can result in a high success and a reasonably low complication rate.

The major complication rate in our CTO PCI series was low (17 of 1036 patients, $1.7 \%$ ), with low mortality ( 3 of 1036 patients, $0.3 \%$ ). This is consistent with a recent weighted meta-analysis of 18,061 patients from 65 studies showing low (and decreasing over time) CTO PCI complication rates (32). However, to maximize safety, adequate training and bail 
out equipment availability is essential for all operators and catheterization laboratories performing CTO PCI (33).

Our study has important limitations. First, although it is the largest US series of its kind reported to date, data from more patients and sites would further add to the generalizability of our findings. Second, efficiently applying a hybrid approach to CTO PCI requires extensive training and expertise in all three major CTO crossing strategies (antegrade wire escalation, antegrade dissection/re-entry, and retrograde), which in turn necessitates both didactic, but most importantly, practical experience $(23,34)$. All cases in our series were performed by seasoned CTO PCI operators in the setting of established CTO PCI programs. Third, successful execution of a hybrid approach requires significant preparation prior to the start of the PCI, such as studying the case obtaining appropriate equipment, and planning for subsequent steps should the initial crossing attempt fail. Moreover, the decision to switch from one crossing approach to another is heavily dependent on the lack for progress with the initially selected technique and on operator technique and judgment (35). Excessive persistence in the face of minimal progress increases the chances for procedural failure due to utilization of limited resources (radiation, contrast, time). In most cases we recommend that no more than 5-10 min should be spent in a stagnant mode without minor (such as reshaping the wire tip or changing to a wire with significantly different properties), or major (such as switching from an antegrade to a retrograde approach) technique adjustments (23). Fourth, the present study focused on acute procedural outcomes and did not evaluate longterm events after CTO PCI. Fifth, central adjudication of the clinical outcomes and core angiographic laboratory analyses were not performed.

In conclusion, our study demonstrates that application of a hybrid approach by targeted use of all available CTO crossing techniques yields high success and low complication rates among various patient populations, operators, and hospitals, supporting its expanded use in CTO PCI.

\section{Acknowledgments}

We would like to thank Katrina L. Mishoe, BS, Anna Kalynych, MD, Harold Carlson, MD, James Lee, MD, Vishal G. Patel, MD, Bavana V. Rangan, BDS, MPH, Michele Roesle, RN, Luke Stone, and Cashel O'Brien for their assistance with the present study.

Study data was collected and managed using REDCap electronic data capture tools hosted at University of Texas Southwestern Medical Center. ${ }^{1}$ REDCap (Research Electronic Data Capture) is a secure, web-based application designed to support data capture for research studies, providing 1) an intuitive interface for validated data entry; 2) audit trails for tracking data manipulation and export procedures; 3) automated export procedures for seamless data downloads to common statistical packages; and 4) procedures for importing data from external sources.

Research reported in this publication was supported by the National Center for Advancing Translational Sciences of the National Institutes of Health" under award Number UL1TR001105. The content is solely the responsibility of the authors and does not necessarily represent the official views of the NIH.

${ }^{1}$ Paul A. Harris, Robert Taylor, Robert Thielke, Jonathon Payne, Nathaniel Gonzalez, Jose G. Conde, Research electronic data capture (REDCap) - A metadata-driven methodology and workflow process for providing translational research informatics support, J Biomed Inform. 2009 Apr;42(2):377-81. 


\section{References}

1. Christakopoulos GE, Christopoulos G, Carlino M, et al. Meta-analysis of Clinical Outcomes of Patients Who Underwent Percutaneous Coronary Interventions for Chronic Total Occlusions. Am J Cardiol. 2015 in press.

2. Stone GW, Reifart NJ, Moussa I, et al. Percutaneous recanalization of chronically occluded coronary arteries: a consensus document: part II. Circulation. 2005; 112:2530-7. [PubMed: 16230504]

3. Carlino M, Magri CJ, Uretsky BF, et al. Treatment of the chronic total occlusion: A call to action for the interventional community. Catheter Cardiovasc Interv. 2015; 85:771-8. [PubMed: 25382239]

4. Strauss BH, Shuvy M, Wijeysundera HC. Revascularization of chronic total occlusions: time to reconsider? J Am Coll Cardiol. 2014; 64:1281-9. [PubMed: 25236522]

5. Sapontis J, Christopoulos G, Grantham JA, et al. Procedural failure of chronic total occlusion percutaneous coronary intervention: Insights from a multicenter US registry. Catheter Cardiovasc Interv. 2015 in press.

6. Galassi A, Grantham JA, Kandzari D, et al. Percutaneous Treatment of Coronary Chronic Total Occlusion. Part 2: Technical Approach. Interventional Cardiology Review. 2014; 9:201-207.

7. Rinfret S, Joyal D, Spratt JC, Buller CE. Chronic total occlusion percutaneous coronary intervention case selection and techniques for the antegrade-only operator. Catheter Cardiovasc Interv. 2015; 85:408-15. [PubMed: 25044668]

8. Michael TT, Papayannis AC, Banerjee S, Brilakis ES. Subintimal dissection/reentry strategies in coronary chronic total occlusion interventions. Circ Cardiovasc Interv. 2012; 5:729-38. [PubMed: 23074346]

9. Colombo A, Mikhail GW, Michev I, et al. Treating chronic total occlusions using subintimal tracking and reentry: the STAR technique. Catheter Cardiovasc Interv. 2005; 64:407-11. [PubMed: 15789384]

10. Whitlow PL, Burke MN, Lombardi WL, et al. Use of a novel crossing and re-entry system in coronary chronic total occlusions that have failed standard crossing techniques: results of the FAST-CTOs (Facilitated Antegrade Steering Technique in Chronic Total Occlusions) trial. JACC Cardiovasc Interv. 2012; 5:393-401. [PubMed: 22516395]

11. Wosik J, Shorrock D, Christopoulos G, et al. Systematic Review of the Bridgepoint System for Crossing Coronary and Peripheral Chronic Total Occlusions. J Invasive Cardiol. 2015 in press.

12. Brilakis ES, Grantham JA, Thompson CA, et al. The retrograde approach to coronary artery chronic total occlusions: a practical approach. Catheter Cardiovasc Interv. 2012; 79:3-19. [PubMed: 22215566]

13. Joyal D, Thompson CA, Grantham JA, Buller CE, Rinfret S. The retrograde technique for recanalization of chronic total occlusions: a step-by-step approach. JACC Cardiovasc Interv. 2012; 5:1-11. [PubMed: 22230144]

14. Karmpaliotis D, Michael TT, Brilakis ES, et al. Retrograde coronary chronic total occlusion revascularization: procedural and in-hospital outcomes from a multicenter registry in the United States. JACC Cardiovasc Interv. 2012; 5:1273-9. [PubMed: 23257376]

15. Galassi AR, Tomasello SD, Reifart N, et al. In-hospital outcomes of percutaneous coronary intervention in patients with chronic total occlusion: insights from the ERCTO (European Registry of Chronic Total Occlusion) registry. EuroIntervention. 2011; 7:472-9. [PubMed: 21764666]

16. Tsuchikane E, Yamane M, Mutoh M, et al. Japanese multicenter registry evaluating the retrograde approach for chronic coronary total occlusion. Catheter Cardiovasc Interv. 2013; 82:E654-61. [PubMed: 23404874]

17. Tsuchikane E, Katoh O, Kimura M, Nasu K, Kinoshita Y, Suzuki T. The first clinical experience with a novel catheter for collateral channel tracking in retrograde approach for chronic coronary total occlusions. JACC Cardiovasc Interv. 2010; 3:165-71. [PubMed: 20170873]

18. Rathore S, Matsuo H, Terashima M, et al. Procedural and in-hospital outcomes after percutaneous coronary intervention for chronic total occlusions of coronary arteries 2002 to 2008: impact of novel guidewire techniques. JACC Cardiovasc Interv. 2009; 2:489-97. [PubMed: 19539251] 
19. Pershad A, Eddin M, Girotra S, Cotugno R, Daniels D, Lombardi W. Validation and incremental value of the hybrid algorithm for CTO PCI. Catheter Cardiovasc Interv. 2014; 84:654-9. [PubMed: 24403122]

20. Brilakis ES, Grantham JA, Rinfret $S$, et al. A percutaneous treatment algorithm for crossing coronary chronic total occlusions. JACC Cardiovasc Interv. 2012; 5:367-79. [PubMed: 22516392]

21. Thompson CA. The Hybrid Approach for Percutaneous Revascularization of Coronary Chronic Total Occlusions. Interventional Cardiology Clinics. 2012; 1:349-353.

22. Garcia S, Abdullah S, Banerjee S, Brilakis ES. Chronic total occlusions: patient selection and overview of advanced techniques. Curr Cardiol Rep. 2013; 15:334. [PubMed: 23319014]

23. Brilakis, ES. A Step-By-Step Approach. Waltham, MA: Elsevier; 2013. Manual of Coronary Chronic Total Occlusion Interventions.

24. El Sabbagh A, Banerjee S, Brilakis ES. Illustration of the 'hybrid' approach to chronic total occlusion crossing. Interventional Cardiology. 2012; 4:639-643.

25. Nombela-Franco L, Urena M, Jerez-Valero M, et al. Validation of the J-chronic total occlusion score for chronic total occlusion percutaneous coronary intervention in an independent contemporary cohort. Circ Cardiovasc Interv. 2013; 6:635-43. [PubMed: 24254710]

26. Michael TT, Mogabgab O, Fuh E, et al. Application of the "hybrid approach" to chronic total occlusion interventions: a detailed procedural analysis. J Interv Cardiol. 2014; 27:36-43. [PubMed: 24456334]

27. Christopoulos G, Menon RV, Karmpaliotis D, et al. The efficacy and safety of the "hybrid" approach to coronary chronic total occlusions: insights from a contemporary multicenter US registry and comparison with prior studies. J Invasive Cardiol. 2014; 26:427-32. [PubMed: 25198485]

28. Saito S. Different strategies of retrograde approach in coronary angioplasty for chronic total occlusion. Catheter Cardiovasc Interv. 2008; 71:8-19. [PubMed: 17985379]

29. Morino Y, Abe M, Morimoto T, et al. Predicting successful guidewire crossing through chronic total occlusion of native coronary lesions within 30 minutes: the J-CTO (Multicenter CTO Registry in Japan) score as a difficulty grading and time assessment tool. JACC Cardiovasc Interv. 2011; 4:213-21. [PubMed: 21349461]

30. Werner GS. The BridgePoint devices to facilitate recanalization of chronic total coronary occlusions through controlled subintimal reentry. Expert Rev Med Devices. 2011; 8:23-9. [PubMed: 21158537]

31. Rathore S, Katoh O, Matsuo H, et al. Retrograde percutaneous recanalization of chronic total occlusion of the coronary arteries: procedural outcomes and predictors of success in contemporary practice. Circ Cardiovasc Interv. 2009; 2:124-32. [PubMed: 20031705]

32. Michael TT, Karmpaliotis D, Brilakis ES, et al. Procedural outcomes of revascularization of chronic total occlusion of native coronary arteries (from a multicenter United States registry). Am J Cardiol. 2013; 112:488-92. [PubMed: 23672987]

33. Patel VG, Brayton KM, Tamayo A, et al. Angiographic success and procedural complications in patients undergoing percutaneous coronary chronic total occlusion interventions: a weighted metaanalysis of 18,061 patients from 65 studies. JACC Cardiovasc Interv. 2013; 6:128-36. [PubMed: 23352817]

34. Brilakis ES, Karmpaliotis D, Patel VG, Banerjee S. Complications of chronic total occlusion angioplasty. Interventional Cardiology Clinics. 2012; 1:373-389.

35. Karmpaliotis D, Lembo N, Kalynych A, et al. Development of a high-volume, multiple-operator program for percutaneous chronic total coronary occlusion revascularization: procedural, clinical, and cost-utilization outcomes. Catheter Cardiovasc Interv. 2013; 82:1-8. [PubMed: 23576529] 


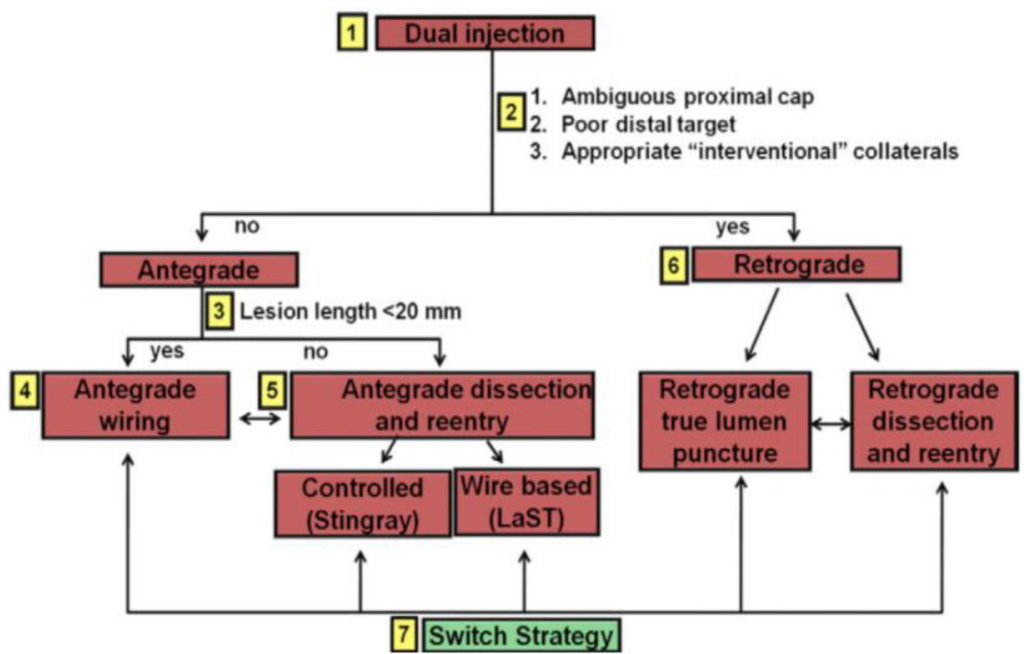

Figure 1.

A hybrid algorithm for CTO crossing (reproduced with permission from reference 20).

The initial crossing strategy selection depends on several angiographic characteristics, such as proximal cap ambiguity, size and calcification of the distal target vessel, lesion length and presence of appropriate collaterals. Strategy switch is recommended if the initially selected strategy fails. 


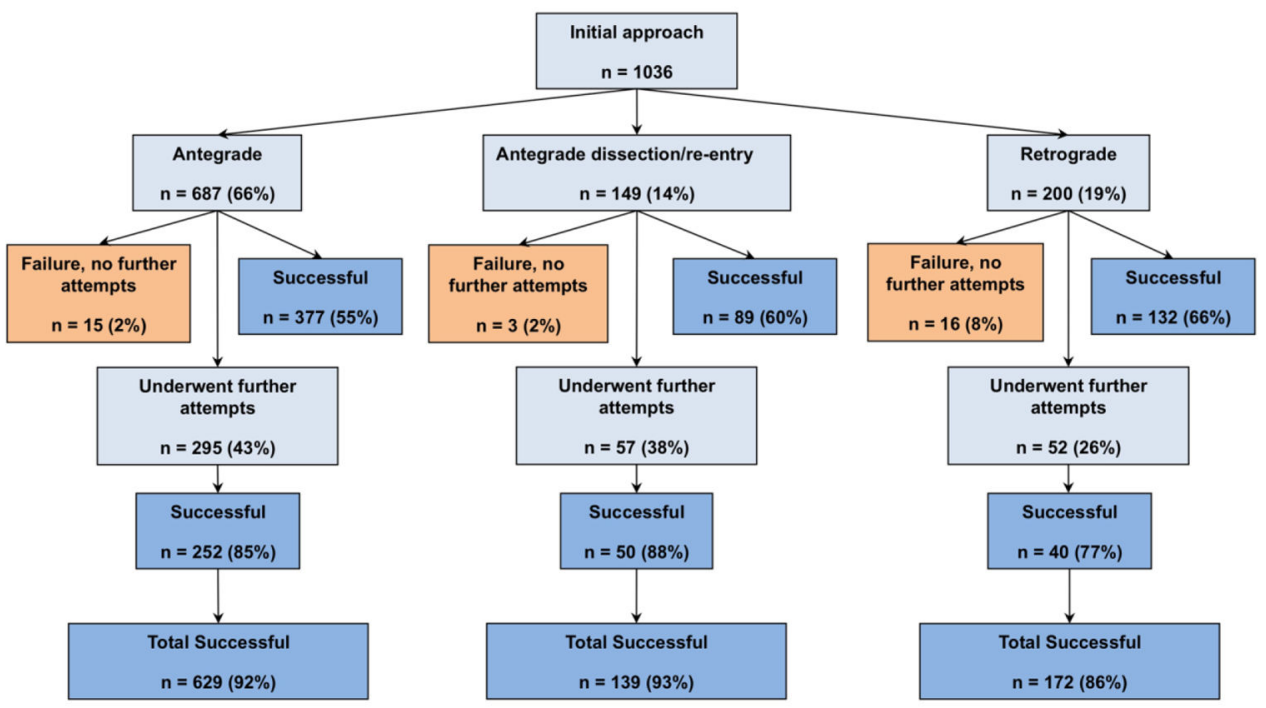

Figure 2.

Flow chart depicting the crossing strategies utilized in the study patients. 


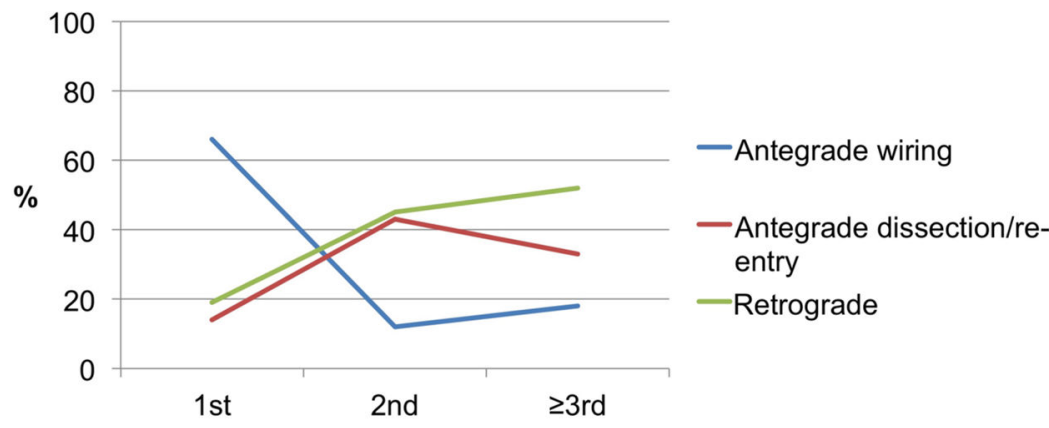

Fig. 3a

Approach sequence
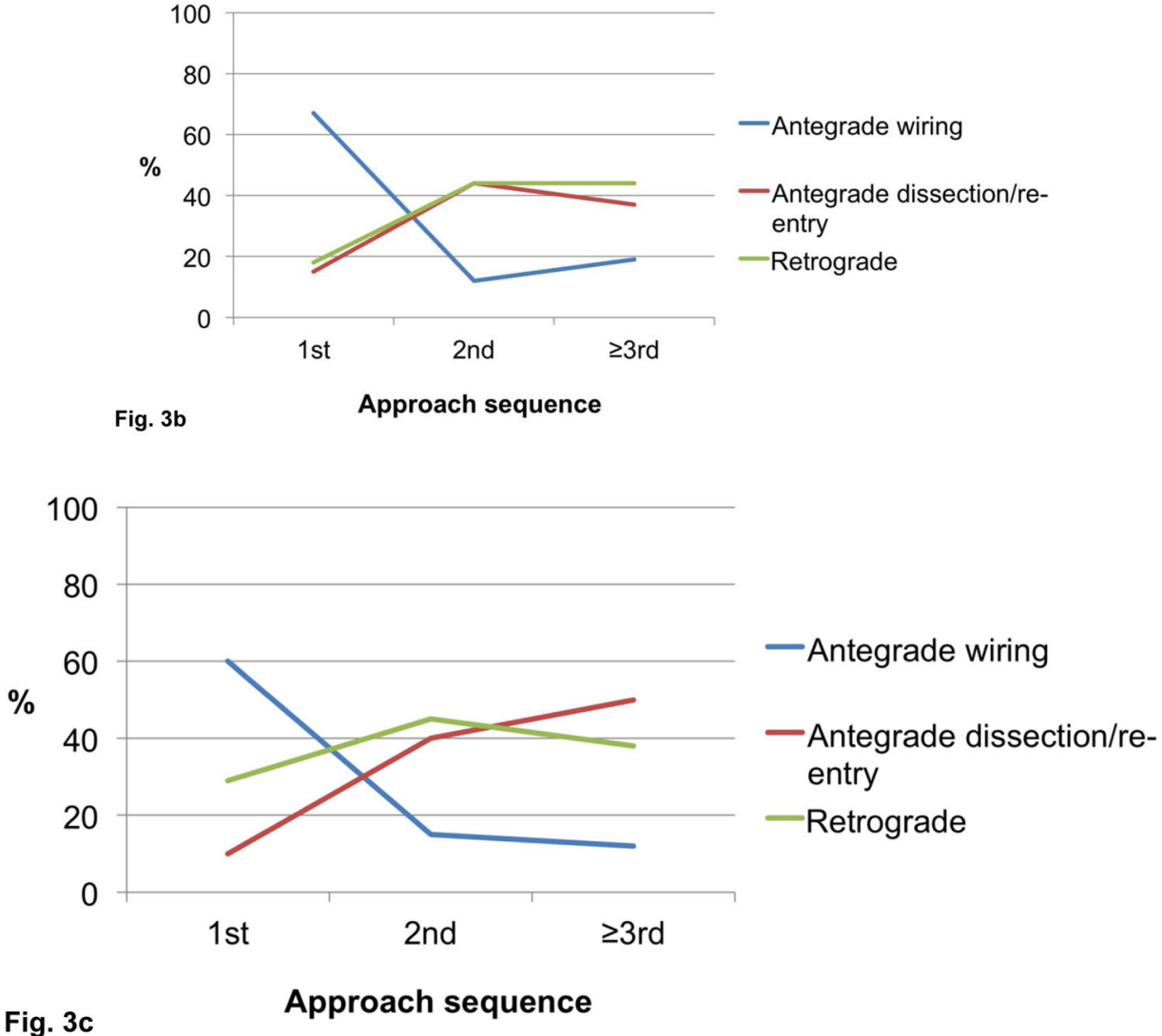

Figure 3.

Utilization of crossing strategies based on crossing sequence in all procedures $(n=1,036$, panel A), successful procedures ( $n=940$, panel B), and failed procedures $(n=96$, panel C). 
Table 1

Clinical and angiographic characteristics of the study patients, classified according to whether technical success was achieved.

\begin{tabular}{|c|c|c|c|c|}
\hline Clinical Characteristics & Overall $(n=1036)$ & Technical Success (n=940) & Technical Failure $(n=96)$ & $p$ \\
\hline Age (years) ${ }^{*}$ & $65 \pm 10$ & $65 \pm 10$ & $67 \pm 10$ & 0.073 \\
\hline Men (\%) & 86 & 85 & 91 & 0.122 \\
\hline Hypertension (\%) & 90 & 89 & 95 & 0.117 \\
\hline Dyslipidemia (\%) & 96 & 95 & 98 & 0.421 \\
\hline Diabetes mellitus (\%) & 43 & 44 & 34 & 0.069 \\
\hline Heart failure (\%) & 28 & 27 & 35 & 0.094 \\
\hline Previous MI (\%) & 42 & 40 & 58 & 0.001 \\
\hline Previous CABG $(\%)$ & 34 & 33 & 46 & 0.013 \\
\hline Previous PCI & 67 & 65 & 78 & 0.015 \\
\hline Previous stroke (\%) & 11 & 11 & 17 & 0.048 \\
\hline Peripheral arterial disease (\%) & 17 & 17 & 24 & 0.078 \\
\hline \multicolumn{5}{|l|}{ Angiographic characteristics } \\
\hline \multicolumn{5}{|l|}{ CTO target vessel } \\
\hline $\mathrm{RCA}(\%)$ & 59 & 58 & 66 & 0.013 \\
\hline $\operatorname{LCX}(\%)$ & 19 & 18 & 24 & 0.015 \\
\hline $\operatorname{LAD}(\%)$ & 23 & 24 & 11 & \\
\hline CTO length $(\mathrm{mm})^{*}$ & $30(20-40)$ & $30(20-40)$ & $38(25-51)$ & 0.014 \\
\hline Moderate/severe calcification (\%) & 56 & 54 & 68 & 0.008 \\
\hline Moderate/severe tortuosity (\%) & 33 & 32 & 45 & 0.010 \\
\hline Prior failed CTO PCI (\%) & 16 & 16 & 19 & 0.401 \\
\hline Lack of "interventional collaterals" (\%) & 39 & 36 & 58 & $<0.001$ \\
\hline In-stent restenosis (\%) & 13 & 12 & 17 & 0.182 \\
\hline J-CTO score ${ }^{*}$ & $2.5 \pm 1.2$ & $2.4 \pm 1.2$ & $3.2 \pm 1.0$ & $<0.001$ \\
\hline
\end{tabular}

CABG, Coronary artery bypass graft surgery; CTO, chronic total occlusion; LAD, left anterior descending artery; LCX, left circumflex artery; MI, myocardial infarction; PCI, percutaneous coronary intervention; RCA, right coronary artery. 


\section{Table 2}

Outcomes among study patients, classified according to whether technical success was achieved or not.

\begin{tabular}{|c|c|c|c|c|}
\hline Outcomes and equipment utilization & Overall $(n=1036)$ & Technical Success $(n=940)$ & Technical Failure $(n=96)$ & $p$ \\
\hline Radial access (\%) & 22 & 21 & 18 & 0.317 \\
\hline Dual injection (\%) & 71 & 69 & 89 & 0.001 \\
\hline Procedural success (\%) & 90 & 99 & 0 & $<0.001$ \\
\hline Procedure time $(\min )^{*}$ & $119(82-175)$ & $115(80-167)$ & $179(127-238)$ & $<0.001$ \\
\hline Fluoroscopy time $(\mathrm{min})^{*}$ & $44(27-72)$ & $41(26-68)$ & $70(49-96)$ & $<0.001$ \\
\hline Patient AK dose (Gray) ${ }^{*}$ & $3.4(2.0-5.4)$ & $3.2(2.0-5.3)$ & $5.3(2.7-7.7)$ & $<0.001$ \\
\hline Patient DAP dose $\left(\text { Gray }^{*} \mathrm{~cm}^{2}\right)^{*}$ & $262(141-397)$ & $258(141-396)$ & $299(140-400)$ & 0.598 \\
\hline Contrast volume $(\mathrm{ml})^{*}$ & $260(200-360)$ & $260(195-350)$ & $305(208-426)$ & 0.005 \\
\hline
\end{tabular}

AK: air kerma; DAP: dose area product. 
Table 3

Crossing techniques utilized in the study patients, classified according to whether or not technical success was achieved or not.

\begin{tabular}{|c|c|c|c|c|}
\hline Crossing technique & Overall $(n=1036)$ & Technical Success (n=940) & Technical Failure $(n=96)$ & $p$ \\
\hline Antegrade wire escalation (\%) & 71 & 71 & 70 & 0.814 \\
\hline Antegrade dissection/re-entry (\%) & 36 & 34 & 49 & 0.005 \\
\hline Retrograde (\%) & 42 & 39 & 66 & $<0.001$ \\
\hline Number of approaches ${ }^{*}$ & $1.5 \pm 0.8$ & $1.5 \pm 0.8$ & $1.9 \pm 0.8$ & $<0.001$ \\
\hline Number of approach changes & $0.5 \pm 0.8$ & $0.5 \pm 0.8$ & $0.9 \pm 0.8$ & $<0.001$ \\
\hline \multicolumn{5}{|l|}{ Initial approach } \\
\hline Antegrade wire escalation $(\%)$ & 66 & 67 & 60 & 0.030 \\
\hline Antegrade dissection/re-entry $(\%)$ & 14 & 15 & 10 & \\
\hline Retrograde $(\%)$ & 19 & 18 & 29 & \\
\hline \multicolumn{5}{|l|}{ Initial approach successful } \\
\hline Overall (\%) & 58 & & & \\
\hline Antegrade wire escalation $(\%)$ & 55 & - & - & - \\
\hline Antegrade dissection/re-entry $(\%)$ & 60 & & & \\
\hline Retrograde (\%) & 66 & & & \\
\hline \multicolumn{5}{|l|}{ Final successful crossing technique } \\
\hline Antegrade wire escalation (\%) & - & 46 & - & - \\
\hline Antegrade dissection/re-entry $(\%)$ & & 26 & & \\
\hline Retrograde $(\%)$ & & 28 & & \\
\hline
\end{tabular}


Table 4

Clinical and angiographic characteristics of the study patients, classified according to initial crossing strategy.

\begin{tabular}{|c|c|c|c|c|}
\hline Variable & $\begin{array}{l}\text { Antegrade wire } \\
\text { escalation }(n=687)\end{array}$ & Antegrade dissection/re-entry ( $n=149)$ & Retrograde $(n=200)$ & $p$ \\
\hline Previous CABG $(\%)$ & 30 & 33 & 50 & $<0.001$ \\
\hline Previous PCI (\%) & 65 & 66 & 72 & 0.175 \\
\hline \multicolumn{5}{|l|}{ CTO target vessel } \\
\hline $\mathrm{RCA}(\%)$ & 53 & 70 & 70 & $<0.001$ \\
\hline $\operatorname{LCX}(\%)$ & 21 & 9 & 17 & \\
\hline $\operatorname{LAD}(\%)$ & 26 & 21 & 13 & \\
\hline Moderate/severe calcification (\%) & 53 & 50 & 67 & 0.001 \\
\hline Moderate/severe tortuosity (\%) & 33 & 27 & 38 & 0.113 \\
\hline Prior failed attempt $(\%)$ & 13 & 22 & 24 & $<0.001$ \\
\hline In-stent restenosis (\%) & 13 & 19 & 9 & 0.023 \\
\hline J-CTO score ${ }^{*}$ & $2.2 \pm 1.2$ & $2.6 \pm 1.2$ & $3.3 \pm 0.9$ & $<0.001$ \\
\hline
\end{tabular}

CABG, Coronary artery bypass graft surgery; CTO, chronic total occlusion; LAD, left anterior descending artery; LCX, left circumflex artery; MI, myocardial infarction; PCI, percutaneous coronary intervention; RCA, right coronary artery. 


\section{Table 5}

Crossing strategies and equipment utilization.

\begin{tabular}{|c|c|}
\hline Successful antegrade wiring $(n=433)$ & \\
\hline \multicolumn{2}{|l|}{ Guidewire that crossed } \\
\hline Pilot 200 & 36 \\
\hline Fielder XT & 19 \\
\hline Fielder FC & 5 \\
\hline Confianza Pro 12 & 11 \\
\hline Runthrough & 8 \\
\hline BMW & 5 \\
\hline Other & 16 \\
\hline \multicolumn{2}{|l|}{ Successful Retrograde $(n=273)$} \\
\hline \multicolumn{2}{|l|}{ Collaterals used ${ }^{*}$} \\
\hline Septal $(\%)$ & 51 \\
\hline Epicardial (\%) & 29 \\
\hline Bypass graft - SVG (\%) & 17 \\
\hline Bypass - LIMA (\%) & 2 \\
\hline \multicolumn{2}{|l|}{ Crossing techniques $*$} \\
\hline Reverse CART (\%) & 67 \\
\hline Retrograde true-to-true lumen (\%) & 19 \\
\hline CART $(\%)$ & 4 \\
\hline Knuckle Wire (\%) & 6 \\
\hline Kissing Wire (\%) & 2 \\
\hline Just marker (\%) & 3 \\
\hline \multicolumn{2}{|c|}{ Successful antegrade dissection/re-entry $(\mathrm{n}=\mathbf{2 4 3})$} \\
\hline Subintimal crossing $(\%)$ & 74 \\
\hline True lumen crossing (\%) & 25 \\
\hline \multicolumn{2}{|l|}{ Successful dissection technique } \\
\hline CrossBoss $(\%)$ & 63 \\
\hline Knuckle Wire (\%) & 28 \\
\hline Antegrade wire entered subintimally (\%) & 9 \\
\hline \multicolumn{2}{|l|}{ Successful re-entry technique } \\
\hline Stingray system $(\%)$ & 79 \\
\hline STAR $(\%)$ & 11 \\
\hline $\operatorname{LAST}(\%)$ & 3 \\
\hline Other $(\%)$ & 7 \\
\hline
\end{tabular}

More than one collaterals or crossing techniques were used in some cases 
CART, controlled antegrade and retrograde tracking and dissection; LAST, limited antegrade subintimal tracking; LIMA, lateral internal mammary artery; STAR, subintimal tracking and re-entry; SVG, saphenous vein graft. 